\title{
Construct, content and face validity of the camera handling trainer (CHT): a new E-BLUS training task for $30^{\circ}$ laparoscope navigation skills
}

\author{
Domenico Veneziano ${ }^{1}$ Andrea Minervini ${ }^{2} \cdot$ John Beatty $^{3} \cdot$ Paolo Fornara $^{4} \cdot$ Ali Gozen $^{5} \cdot$ Francesco Greco $^{6}$. \\ J. F. Langenhuijsen ${ }^{7} \cdot$ Luca Lunelli $^{8} \cdot$ Deirdre Overgaauw $^{9} \cdot$ Jens Rassweiler $^{5} \cdot$ Bernardo Rocco $^{10}$ \\ Rafael Sanchez Salas $^{8} \cdot$ Shahrokh Shariat $^{11} \cdot$ Robert M. Sweet $^{12} \cdot$ Giuseppe Simone $^{13}$ - Christopher Springer ${ }^{4}$. \\ Agostino Tuccio $^{2}$ Ben Van Cleynenbreugel ${ }^{14} \cdot$ Peter Weibl $^{11} \cdot$ Pietro Cozzupoli $^{1}$
}

Received: 23 February 2015 / Accepted: 29 July 2015 / Published online: 6 August 2015

(c) Springer-Verlag Berlin Heidelberg 2015

\begin{abstract}
Purpose Assessing construct, face and content validity of the camera handling trainer (CHT), a novel low-fidelity training device for $30^{\circ}$ laparoscope navigation skills.

Methods We developed a custom-designed box trainer with clinically based graphic targets. A total of 117 participants, stratified according to their previous experience (novice, competent, expert), took part to a CHT session and subsequently were asked to fill out a survey to assess the impact of the $\mathrm{CHT}$ on their $30^{\circ}$ laparoscope navigation skills. Sixty of them were also studied for task performance during a 1-h session, with multiple time measurements.

Results All participants, regardless of the previous experience, significantly improved their performance after the CHT session. Regarding construct validity, the mean task performance on the last measurement for novice group was found to be comparable to the mean first attempt of both
\end{abstract}

Domenico Veneziano

info@domenicoveneziano.it

1 Department of Urology and Renal Transplantation, Bianchi-Melacrino-Morelli Hospital, Via Melacrino No. 1, 89100 Reggio Calabria, Italy

2 Department of Urology, Careggi Hospital, University of Florence, Florence, Italy

3 Department of Urology, Northampton General Hospital NHS Trust, Northampton, UK

4 Department of Urology and Renal Transplantation, MartinLuther-University, Halle/Saale, Germany

5 Department of Urology, Klinikum Heilbronn, Heilbronn, Germany

6 Department of Urology and Mini-invasive Surgery, Romolo Hospital, Rocca di Neto, Italy

7 Department of Urology, Radboud University Nijmegen Medical Centre, Nijmegen, The Netherlands competent $(p=0.12)$ and expert $(p=0.24)$ participants. All participants agreed that "the CHT is a valid training tool" and that "the CHT should be part of the regular dry laboratory training sessions", assessing both face and content validity. Limitations include the need for assessment of predictive validity.

Conclusions The CHT is a valid training tool for $30^{\circ}$ laparoscope navigation and thus should be considered as one of the fundamental exercises during basic laparoscopic hands-on training sessions for urologists.

Keywords $30^{\circ}$ Laparoscopes - Basic laparoscopic skills . Camera handling trainer $\cdot$ E-BLUS $\cdot$ Hands-on training · Laparoscopic camera navigation

8 Department of Urology, Institut Montsouris Paris, Paris, France

9 Wilhelmina Children's Hospital, University Medical Centre Utrecht, Utrecht, The Netherlands

10 Clinica Urologica I, Fondazione IRCCS Ca' Granda Ospedale Maggiore Policlinico, Università degli studi di Milano, Milan, Italy

11 Department of Urology, Medical University of Vienna, Vienna, Austria

12 Department of Urology, SIM Portal, University of Minnesota, Minneapolis, MN, USA

13 Department of Urology, Regina Elena National Cancer Institute, Rome, Italy

14 Department of Urology, University Hospital Gasthuisberg, Katholieke Universiteit Leuven, Louvain, Belgium 


\section{Introduction}

In urology, a laparoscopic skill that is rarely addressed is proper use of the $30^{\circ}$ laparoscope. For visualization of the operative field, surgeons rely daily on camera operators, who are usually the least experienced members of the team. Residents often use a laparoscopic camera for the first time in the operating room (OR) with no previous education or experience [1]. This can lead to sacrificing the additional benefits of an angled laparoscope for the simpler $0^{\circ}$ alternative. According to Fitts and Posner's theory on motor skill acquisition, the earlier stages of learning motor skills can take place outside the OR in a simulation laboratory [2]. Essential skills for camera operators include maintaining a correct orientation, distinguishing the right horizon, having proper zoom control, controlling tremors and using the lens angle properly. These skills are far from being intuitive, and camera operators may need to overcome a considerable learning curve [3]. Camera handling training systems available today on the market present few clinical connections with daily urological practice [4] and are based mostly on expensive virtual reality or camera tracking technologies. On the other side, a wide range of exercises is available to train every instrumental skill needed for a basic proficiency in laparoscopic surgery [5]. Clinically based expertise in tissue manipulation does not automatically entail skilfulness in angled laparoscope navigation, and vice versa [6]. To fill this gap, we created a new low-cost training task for $30^{\circ}$ laparoscopes, specifically designed to simulate real situations of urological practice in a low-fidelity model. Due to its low-fidelity design, the camera handling trainer (CHT) can be used to explain the correct use of any $30^{\circ}$ optics, from laparoscopes to cystoscopes. The aim of the study was to assess construct, face and content validity of the CHT, thus evaluating its future role inside the basic laparoscopic urological curriculum.

\section{Materials and methods}

\section{Equipment}

The CHT has been developed, designed and built by the lead author (D.V.). It is made of three main parts: a Plexiglas viewfinder for objective assessment, a plastic custommade box that contains a graphic path with twelve numbered circles and a specifically designed trocar support with passive feedback (Fig. 1). The construction of a complete CHT unit had a total cost of $70 €$.

For the development of the CHT, an Olympus "endoeye" $30^{\circ}$ laparoscopic camera and a laparoscopic tower with a $28^{\prime \prime}$ flat monitor have been used. The viewfinder had to be anchored over the laparoscopic monitor before the session (Fig. 2). The CHT was designed as a stand-alone training device, with a novel dedicated trocar support. This was made of neoprene and had built-in rubber bands that physically held the trocar and produced "passive feedback". Inside the CHT box, twelve blue numbered circles were placed along a black path.

\section{Camera navigation training method}

The trainee was asked to maintain a correct image of each numbered target within the monitor template for $3 \mathrm{~s}$, as verified via direct observation. On each circle, a line was allowing an objective assessment of horizon control when aligned to the target template. In case of misalignment, the countdown was restarting. The black path indicated the right direction for the camera movements and had to be always visible inside the target template during the session.

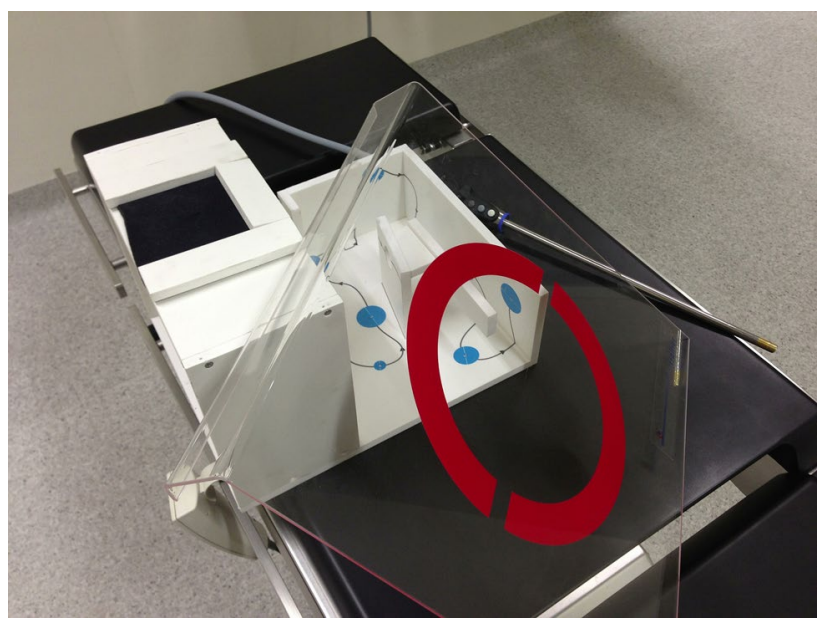

Fig. 1 CHT box with embedded trocar support and viewfinder

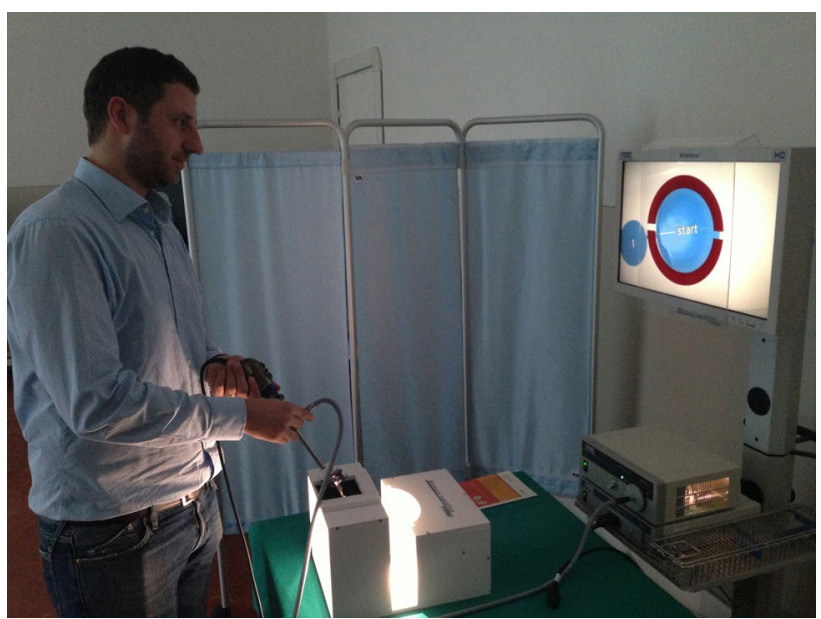

Fig. 2 One of the participants uses the CHT 
During training and testing, each participant acquired circles sequentially following the numerical order. Each circle had a different diameter going from 12 to $40 \mathrm{~mm}$ (Table 1), to force the candidate zooming in and out during the task. To assess the trainees' performance, time to complete the task was measured by a testing proctor. The completion of the whole task was achievable only with a proficient knowledge of camera navigation, and the time needed to perform the different steps of the task decreased when the efficacy of applying this knowledge improved. For this reason, time was considered as a reliable marker to measure performance.

Size and position of the circles were designed to simulate some of the most common situations of urologic laparoscopy (Table 1), thus differentiating the CHT from previous generic laparoscope navigation trainers [4, 7]. Circles from number one to five were placed on the base of the box to force basic zoom in/out and lateral movements. Targeting the smaller circles (numbers two and four) was producing increased tremor due to the fulcrum effect. Number six was on the left side, behind an obstacle, simulating the position achieved when looking behind a left renal hilum, while kidney elevated by traction. Circle number seven was placed on the left upper-lateral part of the box, simulating the position achieved when looking at a lateral wall of the abdomen during a laparoscopy. To target it correctly, the lens had to be turned to the 10 o'clock position. The next circle was placed in the left corner of the box and required full pressure of the laparoscope in the trocar with the lens back to the 6 o'clock position. This circle was placed to mimic the visualization of the upper pole of the right kidney and to remind the participant the importance of repositioning the lens to neutral (6 o'clock) after the previous rotation. Circles numbers nine and ten were placed behind an obstacle. In the middle of the obstacle, a rounded hole was the only pathway to reach the targets, simulating the access to a renal cyst. Once across the obstacle, circle number nine was straight ahead, whereas number ten was placed on the upper side. This required a rotation of the lens to 2 o'clock for a correct visualization. To achieve circle number 11, the laparoscope had to be moved back from the hole, to the right corner of the box, again in neutral position. The 12th circle was placed above the previous target and required a full rotation of the lens. This simulated the action of checking for port bleeding at the end of a procedure.

\section{Validation}

In the present study, we validated the CHT according to the standardized steps of the validation process described by McDougal et al. [8].

Face validity was defined as the "judgment of novices regarding the realism of the simulator and its representation of what it is supposed to represent".

Content validity was defined by experts as "the appropriateness of the simulator as a teaching modality".

Construct validity was the demonstration that experts would outperform novices on the simulator on the basis of their previous experience.

\section{Participants}

A total of 117 participants were enrolled for the study from ten different high-volume laparoscopy centres in Europe. All participants were stratified with auto-assessment, following the proficiency levels described by Dreyfus and Dreyfus: novice (no laparoscopic navigation experience), competent ( $<100$ laparoscopic procedures performed) and expert (more than 100 laparoscopic procedures performed) [9].
Table 1 Description of CHT circles and skills needed to achieve them

\begin{tabular}{lllll}
\hline Circle number & Circle diameter $(\mathrm{cm})$ & Optics position & Simulated clinical situation & Skill required \\
\hline 1 & 2.0 & 6 o'clock & Exploring laparoscopic field & Orientation \\
2 & 1.2 & 6 o'clock & Exploring laparoscopic field & Tremor control \\
3 & 4.0 & 6 o'clock & Exploring laparoscopic field & Zoom control \\
4 & 1.2 & 6 o'clock & Exploring laparoscopic field & Tremor control \\
5 & 3.1 & 6 o'clock & Exploring laparoscopic field & Zoom control \\
6 & 2.1 & 7 o'clock & Behind renal hilum & Angle control \\
7 & 2.5 & 10 o'clock & Abdominal lateral wall & Horizon control \\
8 & 3.0 & 6 o'clock & Right kidney upper pole & Zoom control \\
9 & 3.1 & 6 o'clock & Exploring a cyst & Navigation \\
10 & 3.1 & 2 o'clock & Upper wall of a cyst & Rotation control \\
11 & 2.4 & 6 o'clock & Left kidney upper pole & Zoom control \\
12 & 3.5 & 12 o'clock & Check after trocal removal & Rotation control \\
\hline
\end{tabular}




\section{Data collection}

For the present study, a total of eight CHT units were built. These were provided to each participating centre as needed. All participants had free access to an explanatory video (www.domenicoveneziano.it/camerahandling.wmv) to ensure standardized instructions of the training method. The video was also explaining the assessment method in detail, in order to ensure inter-rater reliability. Previous studies identified that "short camera navigation training sessions could not be considered as sufficient to train novices to competence" [10], so just those who could perform a 1-h training session on the CHT $(60,51.2 \%)$ were selected for task performance analysis. Task performance was measured after three predefined intervals in the session. Trial 1 measured the first attempt after the explanatory video. Trial 2 was given after $40 \mathrm{~min}$ of training. After trial 2, every participant had a further 20-min training before trial 3. The three measurements were used to emphasize the study of learning curve and its plateau, as suggested by Ahmed et al. [11].

At the beginning of every trial, all participants were asked to position the "start" circle in the viewfinder on the monitor. Time measurement started as soon as the optics left the "start" circle and finished after the 3-s count on the last circle (number 12). Between the measured trials, participants could train in all or part of the exercise, depending on their needs. Moreover, they could watch the video as many times as they wanted. After their training sessions, all 117 participants were asked to fill out a survey to evaluate the CHT under different aspects.

\section{Statistical analysis}

Student $t$ test was used to correlate trial performances of participants with different experience, thus to assess construct validity. Regression curves were used to compare average time measurements of subjects. Results are reported as mean \pm SD unless otherwise stated. Statistical significance threshold was set at 0.05 . Face validity and content validity were assessed with Likert scale questionnaires. Statistical analysis was performed using SPSS Statistics (Version 22.0. Armonk, NY: IBM Corp).

\section{Results}

Overall, $61(52.1 \%)$ participants were classified as "novice", 39 (33.3\%) as "competent" and $17(28.4 \%)$ as "expert". Performance measurement, analysed on participants with 60 min of training, showed that on the first trial novices completed the exercise in a mean time of $160.1 \mathrm{~s}$ $( \pm 63.6)$, competent in $119.7 \mathrm{~s}( \pm 48.6)$ and experts in $119.7 \mathrm{~s}( \pm 64.8)$. On the second trial, novices completed
Table 2 Time measurements during the 1-h training session

\begin{tabular}{lccc}
\hline & Trial 1 & Trial 2 & Trial 3 \\
\hline Novice (22) & & & \\
Mean & 160.1 & 121.0 & 98.6 \\
SD & 63.6 & 55.3 & 38.7 \\
Variance & 4044.981 & 3065.048 & 1502.037 \\
Competent (21) & & & \\
Mean & 119.7 & 82.7 & 74.7 \\
SD & 48.6 & 25.5 & 17.5 \\
Variance & 2368.914 & 652.514 & 308.214 \\
Expert (17) & & & \\
Mean & 119.7 & 86.2 & 72.1 \\
SD & 64.8 & 34.6 & 19.8 \\
Variance & 4199.441 & 1198.441 & 395.904 \\
\hline
\end{tabular}

Table 3 Learning curves during the 1-h sessions

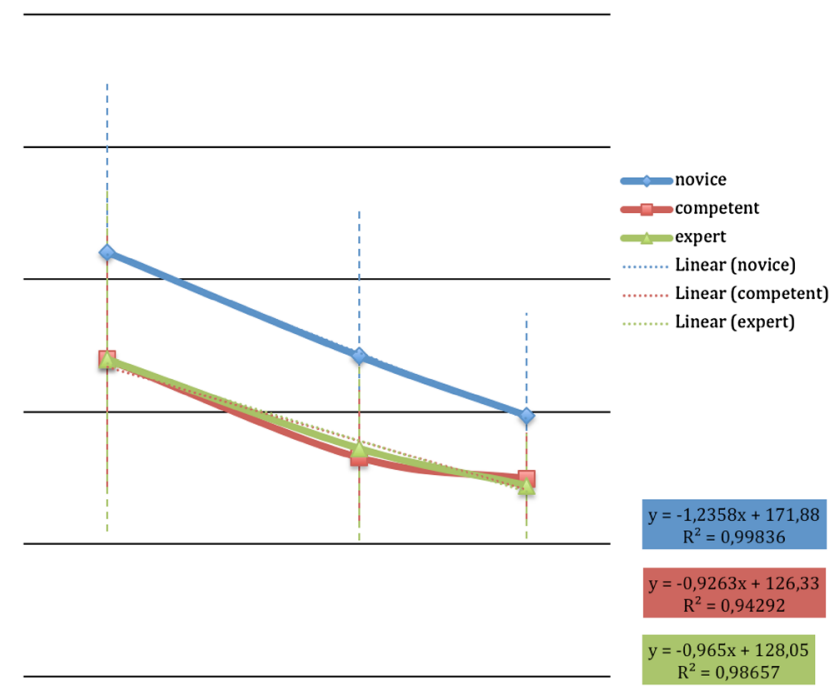

the exercise in a mean of $121 \mathrm{~s}( \pm 55.3)$, competent in $82.7 \mathrm{~s}( \pm 25.5)$ and experts in $86.2 \mathrm{~s}( \pm 34.6)$. On the last trial, novices lowered their mean exercise completion time to $98.6 \mathrm{~s}( \pm 38.7)$, competent reached a mean of $74.7 \mathrm{~s}$ $( \pm 17.5)$, and experts reached $72.1 \mathrm{~s}( \pm 19.8)$ (Table 2$)$.

All participants, despite the different previous experience, improved their performance from trial 1 to trial 3 (novice $p=0.001$, competent $p=0.001$, expert $p=0.009$ ). The mean time of task completion on the last measurement for novice group was not significantly different from the mean first attempt of participants with higher previous experience (versus competent $p=0.12$; versus experts $p=0.24$ ).

Learning curves (Table 3) were similar for all participants with a higher improvement between trials 1 and 2 and a minor but constant increase in performance between trials 2 and $3(p<0.01)$. 
Table 4 Data presented are related to the following scale: 1, strongly disagree; 2 , disagree; 3 , neutral; 4 , agree; 5 , strongly agree

\begin{tabular}{|c|c|c|c|}
\hline & Expert (17) & Competent (39) & Novice (61) \\
\hline \multicolumn{4}{|c|}{ The CHT improved my camera handling skills } \\
\hline Mode & 4 & 5 & 5 \\
\hline Median & 4 & 4 & 5 \\
\hline \multicolumn{4}{|c|}{$\begin{array}{l}\text { The CHT should be required for novices before assisting in the } \\
\text { operating room }\end{array}$} \\
\hline Mode & 5 & 5 & 5 \\
\hline Median & 5 & 5 & 5 \\
\hline \multicolumn{4}{|c|}{ The CHT is useful to understand the full potential of $30^{\circ}$ optics } \\
\hline Mode & 5 & 5 & 5 \\
\hline Median & 5 & 5 & 5 \\
\hline \multicolumn{4}{|c|}{ The CHT is a valid training tool } \\
\hline Mode & 5 & 5 & 5 \\
\hline Median & 5 & 5 & 5 \\
\hline \multicolumn{4}{|c|}{$\begin{array}{l}\text { The CHT should be part of the regular dry laboratory training } \\
\text { sessions }\end{array}$} \\
\hline Mode & 5 & 5 & 5 \\
\hline Median & 5 & 5 & 5 \\
\hline \multicolumn{4}{|c|}{$\begin{array}{l}\text { The CHT explanatory video was clear enough to understand the } \\
\text { exercise }\end{array}$} \\
\hline Mode & 5 & 5 & 5 \\
\hline Median & 5 & 5 & 5 \\
\hline \multicolumn{4}{|c|}{ The CHT trocar support ensures a better training experience } \\
\hline Mode & 5 & 5 & 5 \\
\hline Median & 5 & 5 & 5 \\
\hline
\end{tabular}

Likert scale questionnaires (Table 4) showed homogeneous scores, despite the different experience levels, with the exception of the question pertaining to the perceived improvement in camera handling skills. Every question over 117 survey questionnaires reached a mode and a median of $5 / 5$. The only statement that had overall mode and median of $4 / 5$ was about the perception of experts about personal camera handling skill improvement, after the training session.

\section{Discussion}

Even though poor skills in the handling of laparoscopes can affect the length and the quality of the procedure itself, specific training sessions are not yet provided within the standard basic laparoscopic urological curriculum [5]. A previous study [12] underlined that, being $0^{\circ}$ laparoscope navigation a simpler task, "minimal skill improvement" could be achieved during dedicated training sessions. Meanwhile, the use of $30^{\circ}$ optics requires a specific skill set.

Low-fidelity trainers have proven to be excellent, costeffective resources for basic skill and task training, particularly for novice trainees [13].
Construct validity was assessed by the performance improvement of 60 participants who took part to the 1-h training session, from trial 1 to trial 3. After the explanatory video, while "competent" and "expert" groups were performing similarly with a mean time of $119.7 \mathrm{~s}$ (competent \pm 48.6 ; expert \pm 64.8 ), novices spent a mean time of $160.1 \mathrm{~s}( \pm 63.6)$ to complete the task. While higher variance in SD may be due to the lack of skill for novices, for experts it could be explained by the fact that they are less likely to navigate the camera during their daily practice, even while understanding its rules. This hypothesis is confirmed in trial 2, where "competent" reached a mean completion time of $82.7 \mathrm{~s}( \pm 25.5)$, which was still not significantly different $(p=0.72)$ from the mean performance of experts $(86.2 \mathrm{~s} \pm 34.6)$. These data, in conjunction with a smaller SD, highlight that the "competent" group reached a good confidence with the CHT before experts. This is probably due to the fact that the "competent" group was formed mainly by residents entrusted with the task of holding the camera in the OR. In trial 3, experts had the best mean performance $(72.1 \mathrm{~s} \pm 19.8)$. This result is correlated with their increasing confidence with the training system. After $1 \mathrm{~h}$ of training, novices scored a mean time of $98.6 \mathrm{~s}$ $( \pm 38.7)$, pairing almost the first trial of competent and expert groups and underlining the effectiveness of training on the CHT. Face and content validity was assessed by the Likert scale questionnaires. All participants were satisfied with the explanatory video that was considered "clear enough to understand the exercise". This statement highlighted the value of videos for a standardized introduction to hands-on training tasks. Novices strongly agreed that the CHT improved their camera handling skills. "Expert" and "competent" groups gave a slightly lower score (Table 4) to the same statement, probably in consideration of their previously established knowledge about the rules of camera navigation. This statement would also justify even more the constant improvement of novices through all the three trials, which could be due to the acquisition of both cognitive and practical skills during the exercise. All participants rated with a mode of 5 all of the other questions, adding more validity evidence to the CHT. Experts assessed content validity when agreeing that novices should have a training session on the CHT before assisting in the OR (mode 5). They were also in favour of adding this task to regular dry laboratory training sessions (mode 5). Completion time was measured only in relation to 60 participants who could practice for $1 \mathrm{~h}$ on the CHT. The use of a pre-recorded video explanation of the task avoided any bias related to the experience of the tutor. Moreover, the presence of a dedicated trocar retaining system made the CHT actually portable, with no need for additional box trainers. Its compatibility with any laparoscopic tower and the low production costs make it the ideal device to be 
used in multiple centres for basic laparoscope navigation training. Indeed, the CHT is not an organic-based device and can be used with the regular surgical equipment outside the clinical sessions, thus not requiring the purchase of a dedicated training tower. Moreover, the low-fidelity design allowed for its use as a teaching tool not only for laparoscopic navigation, but also for different kinds of $30^{\circ}$ optics, such as cystoscopes. Every target could be indeed used to remind different areas of the bladder.

A limitation to be listed is that the study was designed to be observational, in order to analyse how participants' skills would react to our novel device, thus lacking of a predetermined sample size for power calculation. Furthermore, no validated questionnaire was already published on the $30^{\circ}$ optics navigation topic, which forced us to formulate a specific Likert scale questionnaire.

Further studies will analyse concurrent validity [8] (comparison between the device and the old technique used for the same training purpose) and predictive validity [8] (whether or not the navigation skills acquired can improve trainee's performance in the operative room) as previously demonstrated by a few studies in relation to more complex and expensive systems $[1,3,14,15]$. In consideration of the references to clinical practice adopted in the development of the CHT (Table 1), the use of a two-arm prospective study could provide additional validity evidence to this training device.

\section{Conclusions}

This study assessed construct, face and content validity of the CHT, a novel low-cost camera navigation system, expressively developed for urological training with $30^{\circ}$ laparoscope.

Taking into account the importance of mastering the use of $30^{\circ}$ laparoscopes for daily practice, the CHT can be considered as a widely reproducible exercise to be included in basic laparoscopic urological "hands-on" training sessions.

Acknowledgments Thanks to Olympus Europe and to the ESU training research group for the kind support offered during the study. Thanks to the "Studio Cassone" in Reggio Calabria for the spaces offered during development phases.

Authors contribution Domenico Veneziano: protocol/project development and manuscript writing/editing; Andrea Minervini: manuscript editing; John Beatty: data collection or management; Paolo Fornara: data collection or management; Ali Gozen: data collection or management; Francesco Greco: data collection or management; J. F. Langenhuijsen: data collection or management; Luca Lunelli: data collection or management; Deirdre Overgaauw: data collection or management; Jens Rassweiler: manuscript editing; Bernardo Rocco: data analysis; Rafael Sanchez Salas: data collection or management; Shahrokh Shariat: data collection or management; Robert M. Sweet: manuscript editing; Giuseppe Simone: data analysis; Christopher
Springer: data collection or management; Agostino Tuccio: data collection or management; Ben Van Cleynenbreugel: manuscript editing; Peter Weibl: data collection or management and Pietro Cozzupoli: manuscript editing.

\section{Compliance with ethical standards}

Conflict of interest The authors declare that they have no conflict of interest.

Ethical standards The manuscript does not contain clinical studies or patient data.

\section{References}

1. Franzeck FM, Rosenthal R, Muller KM, Nocito A, Wittich F, Maurus C et al (2012) Prospective randomized controlled trial of simulator-based versus traditional in-surgery laparoscopic camera navigation training. Surg Endosc 26:235-241

2. Fitts PM, Posner MI (1967) Human performance. Brooks Cole, Belmont

3. Stefanidis D, Haluck R, Pham T, Dunne B, Reinke T, Markley $S$ et al (2007) Construct and face validity and task workload for laparoscopic camera navigation: virtual reality versus video trainer systems at the SAGES Learning Center. Surg Endosc 21:1158-1164

4. Korndorffer R, Hayes DJ, Dunne JB, Sierra R, Touchard CL, Markert RJ et al (2005) Development and transferability of a cost-effective laparoscopic camera navigation simulator. Surg Endosc 19:161-167

5. Brinkman WM, Tjiam IM, Schout BMA, Muijtjens AMM, Van Cleynenbreugel B, Koldewijn EL et al (2014) Results of the European basic laparoscopic urological skills examination. Eur Urol 65:490-496

6. Buzink SN, Botden SMBI, Heemskerk J, Goossens RHM, de Ridder H, Jakimowicz JJ (2009) Camera navigation and tissue manipulation; are those laparoscopic skills related? Surg Endosc 23:750-757

7. Yee KA, Karmali S, Sherman V (2009) Validation of a simple camera navigation trainer. J Am Coll Surg 209:6

8. McDougall EM (2007) Validation of surgical simulators. J Endourol 21(3):244-247

9. Dreyfus HL, Dreyfus SE (1986) Mind over machine: the power of human intuition and expertise in the era of the computer. The Free Press, New York

10. Buzink SN, Goossens RHM, de Ridder H, Jakimowicz JJ (2010) Training of basic laparoscopy skills on SimSurgery SEP. Minim Invasive Ther 19:35-41

11. Ahmed K, Jawad M, Abboudi M, Gavazzi A, Darzi A, Athanasiou $\mathrm{T}$ et al (2011) Effectiveness of procedural simulation in urology: a systematic review. J Urol 186:26-34

12. Shetty S, Panait L, Baranoski J, Dudrick SJ, Bell RL, Roberts $\mathrm{KE}$ et al (2012) Construct and face validity of a virtual realitybased camera navigation curriculum. J Surg Res 177:191-195

13. Wignall GR, Denstedt JD, Preminger GM, Cadeddu JA, Pearle MS, Sweet RM et al (2008) Surgical simulation: a urological perspective. J Urol 179:1690-1699

14. Andreatta PB, Woodrum DT, Birkmeyer JD, Yellamanchilli RK, Doherty GM, Gauger PG et al (2006) Laparoscopic skills are improved with LapMentor training: results of a randomized, double-blinded study. Ann Surg 243:854-860

15. Ganai S, Donroe JA, Louis MRS, Lewis GM, Seymour NE (2007) Virtual-reality training improves angled telescope skills in novice laparoscopists. Am J Surg 193:260-265 